\title{
El ramipril reduce significativamente la mortalidad y los eventos vas- culares en pacientes de alto riesgo
}

Effect of an angiotensin -converting-enzyme inhibitor, ramipril, on cardiovascular events in high risk patients. The Heart Outcomes Prevention Evaluation Study Investigators. N Engl J Med 2000; 342;145-153.

\section{Objetivo}

Evaluar los efectos de un inhibidor de la enzima convertidora de angiotensina (IECA), ramipril, en la prevención de eventos cardiovasculares en una población de alto riesgo.

\section{Diseño}

Estudio factorial $(2 \times 2)^{*}$, multicéntrico, aleatorizado, doble ciego, controlado contra placebo.

\section{Lugar}

Estudio multicéntrico internacional, realizado en 267 centros: 129 de Canadá, 27 de EE.UU., 76 de Europa Occidental, 14 de Argentina y Brasil y 5 de México.

\section{Pacientes}

Se incluyeron 9541 pacientes $>55$ años con evidencia de enfermedad vascular (enfermedad coronaria, accidente cerebrovascular, enfermedad vascular periférica o diabetes más otro factor de riesgo cardiovascular). Se excluyeron aquellos con insuficiencia cardíaca, una fracción de eyección reducida $(<40 \%)$, tratamiento previo con vitamina E o IECA o eventos agudos las 4 semanas previas.

\section{Intervención}

Luego de una fase inicial (run-in*) se aleatorizóa los pacientes a recibir ramipril (10 mg/día, a alcanzar en 4 semanas) o placebo y vitamina E400UI/día o placebo (diseño $2 \times 2$ factorial), con un seguimiento medio de 5 años.

\section{Medición de los Resultados Principales}

El punto final primario fue la combinación de muerte cardiovascular , infarto de miocardio y accidente cerebrovascular. Los puntos finales secundarios incluyeron separadamente cada componente del punto pri- mario, revascularización, desarrollo de insuficiencia cardíaca o angina inestable y complicaciones de la diabetes.

\section{Resultados Principales}

El estudio terminó prematuramente a los 4,5 años de seguimiento debido al beneficio definitivo observado en la rama de pacientes asignados a tratamiento con ramipril.

El tratamiento con ramiprilse asoció con un beneficio clínicamente relevante tanto en el punto primario como en los secundarios (ver tabla). Además se detectó un efecto beneficioso en el desarrollo de complicaciones de la diabetes ( $6,4 \%$ vs. 7,6 ; RR 0,84 IC95\% 0,72-0,98; $p=0,03$ ) y sobre el requerimiento de procedimientos de revascularización ( $16 \%$ vs. $18,4 \%$ RR 0,85 IC $95 \% 0 ; 77-0,94 ; p=0,002)$. No existió efecto favorable en la hospitalización por angina inestable o insuficiencia cardíaca. Si bien se observó beneficio en la mortalidad por todas las causas, no se objetivó efecto en la mortalidad no cardiovascular La diferencia de las tasas del punto final primario se comenzaron a evidenciar a partir del primer año de seguimiento y las curvas de sobrevida continuaron separándose hasta el final del seguimiento ( 4 años). El beneficio con ramipril fue consistente en todos los principales subgrupos.

\begin{tabular}{lcclc}
\hline Evento & Ramipril & Placebo & \multicolumn{1}{c}{ RR [IC95\%] } & NNT \\
\hline Primario & $14 \%$ & $17,8 \%$ & $0,78[0,7,0,86]$ & 27 \\
\hline Muerte CV & $6,1 \%$ & $8,1 \%$ & $0,74[0,64,0,87]$ & 50 \\
\hline IAM & $9,9 \%$ & $12,3 \%$ & $0,80[0,7,0,9]$ & 42 \\
\hline ACV & $3,4 \%$ & $4,9 \%$ & $0,68[0,57,0,79]$ & 67 \\
\hline $\begin{array}{l}\text { Mortalidad } \\
\text { global }\end{array}$ & $10,4 \%$ & $12,2 \%$ & $0,84[0,75,0,95]$ & 56 \\
\hline
\end{tabular}

Conclusiones

El tratamiento con $10 \mathrm{mg} /$ día de ramipril reduce significativamente la ocurrencia de muerte, infarto de miocardio y accidente cerebrovascuLar en pacientes de alto riesgo que no presentan baja fracción de eyección o insuficiencia cardíaca.

Fuente de financiamiento: Medical Research Council of Canada, Heart and Stroke Foundation of Ontario y Laboratorios farmacéuticos productores de Ramipril y Vitamina E.

\section{COMENTARIO}

Datos epidemiológicos y experimentales habían sugerido que la activación del sistema renina-angiotensina presentaba un importante rol en el incremento del riesgo de los eventos cardiovasculares. En un reciente metaanálisis de 3 estudios $^{1-3}$ que incluyó más de 9000 pacientes con fracción de eyección reducida, el tratamiento con IECA redujo el riesgo de infarto de miocardio un $23 \%$. A su vez este beneficio fue independiente de la fracción de eyección, causa de la enfermedad cardíaca, uso concomitante de medicaciones, estado diabético y presión arterial. Sin embargo, esta extensión del beneficio no fue ampliamente aceptada.

En este estudio el tratamiento con ramipril demostró un benefició clínico relevante en pacientes de alto riesgo con fracción de eyección normal o sin historia de insuficiencia cardíaca, que se extiende a todos los subgrupos estudiados y que comienza a manifestarse a partir del primer año de seguimiento: tratando 1000 pacientes con ramipril durante 4 años se previenen 150 eventos en 70 pacientes.

La rápida y sostenida respuesta al ramiprily la continua divergencia con los resultados del grupo placebo sugieren que el tratamiento a largo plazo podría ser efectivo .

*Ver Glosario

\section{Dr. Rodolfo Pizarro}

Servicio de Cardiología. Hospital Italiano de Buenos Aires.

\section{Referencias}

1. Yusuf S, Pepine CJ, Garces, y col. Effect of enalapril on myocardial infarction and unstable angina in patients with low eyection fraction.Lancet 1992; 340; $1173-1178$.

2. Pfeffer MA, Braunwald E, Moyé LA, y col. Effect of captopril on mortality and morbidity in patients with left ventricular dysfunction after myocardial infarction: Resukts of the Survival and Ventricur lar Enlargement Trial. N Engl J Med 1992; 327; 669-677.

3. Lonn EM, YusufS, Jha P, y col. Emerging role of angiotensin converting enzyme inhibitors in cardiac and vascular protection. Circulation 1994; $90 ; 2056-2069$.

4. Tung Ch J, Mark D. Cost- effectiveness in cardiovascular prevention. In Yusuf S, Cairns JA, Camm AJ, y col. Evidence based cardiology. London : BMJ Books; $1998 ; 303-315$.

5. Antiplatelets Trialists Collaboration. Collaborative overview of randomised trials of antiplatelet therapy. Prevention of death, myocardial infarction, and stroke by prolonged antiplatelet therapy in various categories of patients. BMJ1994; 308; 81-106.

6. The SOLVD Investigators. Effect of enalapril on mortality and the development of heart failure in asymptomatic patients with reduced left ventricular eyection fraction. Nengl $\mathrm{J}$ Med $1992 ; 327 ; 685-691$.

7. Kober L, Torp- Pedersen C, Carlsen JE, y col. A clinical trial of trandopril in patients with Left ventricular dysfunction after myocardial infarction. N Engl J Med 1995; 333; $1670-1676$.

8. The Heart Outcomes Prevention Evaluation Study Investigators. Vitamin E supplementation and cardiovascular events in high risk patients N Engl J Med 2000; $342 ; 154-160$. 